\title{
Efecto del tipo de acero y su acabado superficial sobre la cinética de fosfatizado con manganeso
}

\section{Effect of the type of steel and its surface finish on the kinetics of phosphatized with manganese}

GÓMEZ-MACÍAS, Josué†’, CONEJO-FLORES, Ricardo’, GARCÍA-GONZÁLEZ, Juan Manuel** y FUENTES-ACEITUNO, Juan Carlos""

\begin{abstract}
Universidad Autónoma de Zacatecas. Unidad Académica de Ciencias Químicas, Programa Académico de Ingeniería Química, Campus UAZ Siglo XXI. Carretera Zacatecas - Guadalajara Km. 6, Ejido la Escondida, 98160 Zacatecas, Zac. México.

“CINVESTAV-Saltillo, Av. Industria Metalúrgica No. 1062, Parque Industrial Ramos Arizpe, Ramos Arizpe, Coahuila. C.P. 25900, México.
\end{abstract}

ID 1 ${ }^{\text {er }}$ Autor: Josúe, Gómez-Macías / ORC ID: 0000-0003-3180-3714, CVU CONACYT ID: 853102

ID $1^{\text {er }}$ Coautor: Ricardo, Conejo-Flores / ORC ID: 0000-0002-8513-1821, CVU CONACYT ID: 518968

ID $2^{\text {do }}$ Coautor: Juan Manuel, García-González / ORC ID: 0000-0001-7259-5021, CVU CONACYT ID: 346241

ID $3^{\text {er }}$ Coautor: Juan Carlos, Fuentes-Aceituno / ORC ID: 0000-0002-3566-2835, CVU CONACYT ID: 160396

DOI: $10.35429 /$ JOIE.2020.15.4.1.17

Recibido Julio 10, 2020; Aceptado Diciembre 30, 2020

\begin{abstract}
Resumen
El objetivo de la presente investigación, es estudiar el proceso de fosfatizado con manganeso por inmersión. En la literatura no existe información suficiente al proceso de fosfatizado con fosfato de manganeso, ahí la principal aportación del presente objeto de estudio. Desde el marco metodológico se involucra un estudio electroquímico, mediante técnicas de potencial de circuito abierto con el fin de seguir la cinética del proceso. De igual forma se realizó la caracterización del recubrimiento formado sobre la superficie de cada acero utilizado, empleando microscopía electrónica de barrido y espectroscopía por dispersión de energía de Rayos-X. A través de esta técnica se encontró el tiempo en el cual se desarrolló la capa interna del recubrimiento en los sustratos fosfatizados, tanto en las caras desbastadas como en las caras no desbastadas de las muestras de acero. Los resultados que se obtienen muestran que la formación del recubrimiento de fosfatos de manganeso en los aceros depende tanto del tamaño de grano de los aceros en un inicio del proceso, así como del acabado superficial al cual sea sometido la superficie del acero, dando como resultado un incremento del tamaño de los cristales, y afectando la velocidad de desintegración y crecimiento de los mismos.
\end{abstract}

\begin{abstract}
The objective of this work is to study the process of phosphatizing with manganese by immersion. In the literature there is not enough information on the phosphate process with manganese phosphate, hence the main contribution of the present. The methodology involves an electrochemical study, using open circuit potential techniques in order to follow the kinetics of the process. In the same way, the characterization of the coating formed on the surface of each steel used was carried out using scanning electron microscopy and X-ray energy dispersion spectroscopy. Through this technique, the time was found in which the internal layer of the coating developed on the phosphatized substrates, both on the rough and the rough sides of the steel samples. The results obtained show that the formation of the manganese phosphate coating on the steels depends both on the grain size of the steels at the beginning of the process, as well as on the surface finish to which the steel surface is subjected, resulting in an increase in the size of the crystals, and affecting the rate of disintegration and growth of the same.
\end{abstract} Revista. Revista de Ingeniería Innovativa. 2020. 4-15:1-17.

\footnotetext{
* Correspondencia del Autor (jmgarcia@uaz.edu.mx)

$\dagger$ Investigador contribuyendo como primer autor.
} 


\section{Introducción}

El acero es un tipo de aleación férrea conformada principalmente de hierro-carbono. Es uno de los materiales estructurales primordiales para el desarrollo de la vida humana, presentando un mayor interés en el área de la construcción, fabricación de automóviles y línea blanca. Para que el acero presente una alta resistencia a la deformación mecánica, alta dureza (resistencia a la penetración), bajo desgaste por fricción, resistencia a la corrosión, entre otros, éste debe ser sometido a procesos químicos o físicos para que adquiera todas o ciertas características que van de acuerdo a la aplicación que se le confiera [1].

Los tratamientos para el recubrimiento por conversión química son el cromado, oxalatizado y el fosfatizado, al ser el fosfatizado uno de los procesos más empleados en la industria, además es aplicable en superficies ferrosas o no ferrosas. Existen diferentes tipos de fosfatizado: zinc, hierro y manganeso, estos recubrimientos se pueden aplicar en la superficie del sustrato por medio de dos procesos: aspersión e inmersión en los que se involucran, etapas previas y posteriores al fosfatizado como desengrasado, decapado, activación, enjuagues intermedios, entre otros [2].

$\mathrm{Su}$ uso habitual es en la industria automotriz como base de pinturas para incrementar la protección a la corrosión y en la industria petrolera. Todo esto debido al aspecto económico, así como la velocidad de operación y la habilidad de aumentar la resistencia ante la corrosión del material, resistencia al desgaste adhesivo y aumentando la lubricación y la adhesión de películas posteriores al tratamiento [3].

Todas las soluciones convencionales de fosfatizado son soluciones de uno o más metales alcalinos disueltos en ácido fosfórico $\left(\mathrm{H}_{3} \mathrm{PO}_{4}\right)$. En esencia, estas soluciones contienen ácido fosfórico libre y fosfatos primarios de los iones del metal contenido en el baño. Las tres series de sales de fosfato que pueden formarse con un metal univalente (Me) son las siguientes: $\mathrm{MeH}_{2} \mathrm{PO}_{4}$ primario, $\mathrm{Me}_{2} \mathrm{HPO}_{4}$ secundario y el $\mathrm{Me}_{3} \mathrm{PO}_{4}$ terciario [4].
López (2008), explora el efecto que tienen las sales de titanio en las características de los recubrimientos de fosfato mediante estudios de distribución del agente activador y de los cristales de fosfato. Pudo distinguir, mediante el seguimiento de la evolución del tamaño de los cristales con el tiempo, que el proceso de formación de los recubrimientos contempla etapas de nucleación, crecimiento, traslape y redisolución de los cristales de fosfato a lo largo del proceso [5]. Cruz (2010), realizó fosfatizado de cinc y manganeso sobre acero 304 sensibilizado, recupera y demuestra que se incrementa en forma considerable la adherencia entre el sustrato y el recubrimiento anticorrosivo [6]. Onofre et al. (2010), aplica un proceso por inmersión de placas de acero AISI 1010 bajo el empleo de una solución fosfatante comercial (ácido fosfórico y sulfato de manganeso).

Generaron una película de conversión de 1 a $2 \mu \mathrm{m}$, con un incremento a la resistencia a la corrosión [7]. Alvarado (2017), Llevó a cabo un estudio del proceso de fosfatizado electroquímico de acero AISI SAE 1018, mediante las técnicas electroquímicas de voltamperometría cíclica (VC) y cronoamperometría (CA).

Determinó que la respuesta cronoamperométrica corresponde a una suma de 4 contribuciones, relacionadas con la adsorción de iones fosfato, disolución de $\mathrm{Fe}$, crecimiento 2D de una película conductora de $\mathrm{FePO}_{4}$ (s) y el crecimiento $3 \mathrm{D}$ de esta misma película con características menos conductoras [8]. Calle (2019), estudió el proceso de fosfatizado por inmersión en caliente para determinar la masa de fosfato de zinc depositado sobre la superficie de placas de acero en el que se trabajó con concentraciones de la solución de 4,5 y $6 \%$ v/v, temperaturas del baño de 60,70 y $80{ }^{\circ} \mathrm{C}$ y tiempos de inmersión de 60, 120 y 180 s [9]. Romero et al (2019), demostraron la efectividad de las pinturas de poliuretano para la protección contra la corrosión del acero galvanizado [10].

Menor de Gaspar (2020), determinó la influencia del método de corte en las propiedades de tracción al hacer agujeros en componentes de acero destinados a uniones atornilladas [11]. Flores (2020), analizó la precipitación y el engrosamiento de la fase cementita en un acero ultrabajo carbono POSCO CWA0802 [12]. 
Madariaga et al (2020), evaluaron los efectos causados por la aplicación de flux de $\mathrm{CaCO}_{3}$ y $\mathrm{SiO}_{2}$ sobre la morfología del cordón, los parámetros operacionales y la estabilidad del proceso, en unas soldaduras GMAW aplicadas sobre un acero AISI/SAE 1020 [13]. Fernández et al (2020), analizaron el efecto del calor producto de la soldadura en las variaciones microestructurales de una unión disímil de acero endurecible por precipitación, del tipo 17-4 PH (AISI 630) y la superaleación Hastelloy C-276 (B 574) [14].

\section{Mecanismo de fosfatizado}

Una de las más grandes aportaciones al estado del conocimiento para aclarar el mecanismo del fosfatizado la realizo W. Machu [15], él fue el primero en considerar el fosfatizado como un proceso electroquímico y topoquímico. Debido a este enfoque se encontró un gran avance para explicar la cinética del proceso, el efecto de acelerantes de las reacciones de oxidación y reducción y la influencia de tratamientos en la superficie metálica previos al fosfatizado.

Otros investigadores asumen que la precipitación de los fosfatos insolubles se produce en regiones anódicas del metal debido a la importante concentración de iones metálicos en la zona anódica, mientras que Machu y otros autores sostienen que la precipitación se produce sólo en las zonas catódicas donde hay un cambio importante de $\mathrm{pH}$ debido a la descarga de iones de hidrógeno [10]. Por otro lado, Krutivov supone que, en las primeras etapas de tratamiento, hay una formación de una película delgada de fosfatos en las zonas anódicas que luego se convierten en zonas catódicas. Por consiguiente, la descarga de los iones de hidrógeno en las zonas catódicas da lugar a la formación de partículas coloidales que conducen a la precipitación de cristales de fosfato en los cátodos [15].

Gilbert supone que, durante el fosfatizado en un baño que contenga nitratos como acelerantes, se puede formar óxido de hierro II (FeO) en la interfase metal-disolución de acuerdo con la siguiente reacción $[14,15]$ :

$$
\begin{aligned}
& \mathrm{Fe}+3 \mathrm{H}^{+}+\mathrm{NO}_{3}^{-} \rightarrow \mathrm{Fe}^{+2}+\mathrm{HNO}_{2}+\mathrm{H}_{2} \mathrm{O} \\
& \mathrm{Fe}+2 \mathrm{HNO}_{2} \rightarrow 2 \mathrm{NO}+\mathrm{H}_{2} \mathrm{O}+\mathrm{FeO}
\end{aligned}
$$

De acuerdo con investigaciones realizadas por Ghali (1972) en el ámbito del fosfatizado de aceros, se encontró que el mecanismo de fosfatizado con zinc se realiza en cuatro etapas: ataque electroquímico del acero, precipitación de amorfa, cristalización y crecimiento y finalmente reorganización cristalina [15]. Cabe mencionar que este mecanismo también ha sido aceptado para el fosfatizado con manganeso [17].

En la primera etapa, la superficie del sustrato es oxidada de $\mathrm{Fe}$ a $\mathrm{Fe} e^{+2}$ para formar un fosfato primario soluble.

$\mathrm{Fe}+2 \mathrm{H}_{3} \mathrm{PO}_{4} \rightarrow \mathrm{Fe}\left(\mathrm{H}_{2} \mathrm{PO}_{4}\right)_{2}+\mathrm{H}_{2} \uparrow$

Además, cualquier óxido de la superficie será disuelto como indica la siguiente reacción [15]:

$\mathrm{FeO}+2 \mathrm{H}_{3} \mathrm{PO}_{4} \rightarrow \mathrm{Fe}\left(\mathrm{H}_{2} \mathrm{PO}_{4}\right)_{2}+\mathrm{H}_{2} \mathrm{O}$

En la segunda etapa, ocurre una precipitación masiva de fosfatos debido al ataque químico en la etapa anterior, este ataque ocasiona un cambio en el equilibrio del baño fosfatizante en la interfase metal-solución. La precipitación de fosfatos sobre la superficie metálica tiene una estructura amorfa [15]. La reacción que ocurre en esta etapa es [4]:

$\left(\mathrm{H}_{2} \mathrm{PO}_{4}\right)_{2} \rightleftarrows \mathrm{FeHPO}_{4}+\mathrm{H}_{3} \mathrm{PO}_{4}$

El agotamiento de iones de hidrógeno causa la precipitación a un fosfato terciario insoluble de acuerdo con la siguiente reacción [4]:

$3 \mathrm{FeHPO}_{4} \rightleftarrows \mathrm{Fe}_{3}\left(\mathrm{PO}_{4}\right)_{2} \downarrow+\mathrm{H}_{3} \mathrm{PO}_{4}$

Se puede indagar en que el ataque sobre el metal no cesa por completo durante este período y que el fenómeno de precipitación se superpone al ataque. Estos fosfatos, de acuerdo a Ghali, conforman una capa primaria rica en hierro que sirve como una base de anclaje para el fosfatizado [15].

En la etapa de cristalización y crecimiento se da la formación de hopeita o hureaulita (dependiendo del constituyente principal del recubrimiento de fosfato), la cual ocurre sobre la capa de fosfatos amorfos. 
Para el caso de fosfato de zinc la precipitación y crecimiento de cristales ocurre en los sitios anódicos debido a la producción de iones metálicos en el ánodo. Además, el ataque electroquímico del sustrato disminuye por el agotamiento de iones metálicos en la región anódica debido a la nucleación de cristales de fosfofilita (fosfato hidratado de hierro y zinc). Conforme los ánodos se cubren con estos cristales, el ataque en el sustrato es más uniforme. Por lo tanto, esta etapa de crecimiento no es controlada por el contenido de iones metálicos ferrosos, sino por el pH local [4].

La reorganización cristalina es un fenómeno de disolución y re-precipitación de la capa de fosfato controlado por el cambio de $\mathrm{pH}$ en la interfase metal-disolución. Durante esta etapa, los fosfatos del recubrimiento se disuelven y se vuelven a precipitar con gran rapidez, esto se traduce en una reorganización cristalina acompañada de un descenso rápido y significativo en la porosidad llegado a ser menor del $1 \%$ [15]. Cuando en el fosfatizado se emplea manganeso para formar un fosfato de manganeso, los cristales varían en la razón $\mathrm{Mn} / \mathrm{Fe}$ manteniendo la estructura hureaulita. Las reacciones que ocurren con el manganeso son las siguientes [15]:

$\mathrm{Mn}+2 \mathrm{H}_{3} \mathrm{PO}_{4} \rightarrow \mathrm{Mn}\left(\mathrm{H}_{2} \mathrm{PO}_{4}\right)_{2}+\mathrm{H}_{2} \uparrow$

$\mathrm{Mn}\left(\mathrm{H}_{2} \mathrm{PO}_{4}\right)_{2} \rightarrow \mathrm{MnHPO}_{4}+\mathrm{H}_{3} \mathrm{PO}_{4}$

El $\mathrm{MnHPO}_{4}$ es ligeramente soluble, al aumentar el pH debido a la reducción del ión hidrógeno, esta especie pierde un átomo de hidrógeno, para finalmente, formar la capa externa de fosfato de manganeso (o zinc en caso de emplear este metal para la fabricación de la solución fosfatizante), $\mathrm{Mn}_{5} \mathrm{H}_{2}\left(\mathrm{PO}_{4}\right)_{4}$, por cualquiera de las siguientes reacciones [4]:

$$
\begin{aligned}
& 5 \mathrm{MnHPO}_{4} \rightarrow \mathrm{Mn}_{5} \mathrm{H}_{2}\left(\mathrm{PO}_{4}\right)_{4}+\mathrm{H}_{3} \mathrm{PO}_{4} \\
& 6 \mathrm{MnHPO}_{4} \rightarrow 2 \mathrm{Mn}_{3}\left(\mathrm{PO}_{4}\right)_{2}+2 \mathrm{H}_{3} \mathrm{PO}_{4} \\
& 2 \mathrm{H}_{3} \mathrm{PO}_{4}+5 \mathrm{Mn}_{3}\left(\mathrm{PO}_{4}\right)_{2} \rightarrow 3 \mathrm{MnSH}_{2}\left(\mathrm{PO}_{4}\right)_{4} \cdot 4 \mathrm{H}_{2} \mathrm{O}
\end{aligned}
$$

En la Figura 1, se esquematiza el mecanismo de fosfatizado.

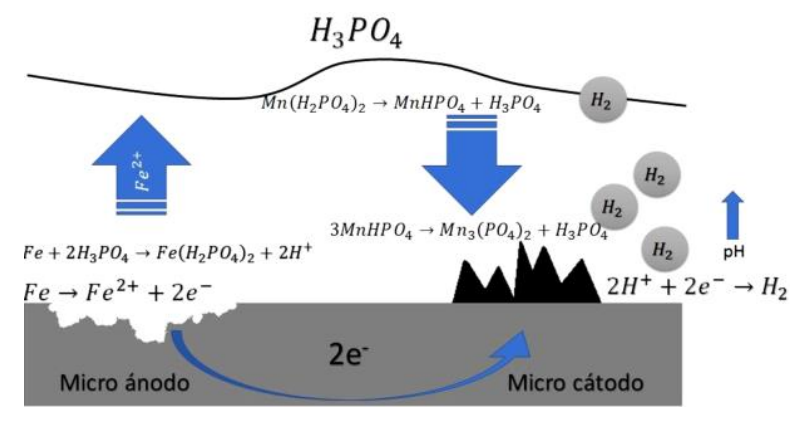

Figura 1 Mecanismo de fosfatizado en solución ácida de manganeso [16]

\section{Mecanismo fenomenológico del fosfatizado}

A diferencia del mecanismo anterior, donde la etapa de reorganización es una etapa final, en el mecanismo moderno la etapa de reorganización cristalina es un proceso cíclico. Este proceso consta de varias etapas y es aplicable para todos los sistemas de fosfatizado que exhiben una capa interna durante el proceso [17], al ser las tres primeras las mismas que propone Ghali [15], las etapas son:

\section{- $\quad$ Ataque o corrosión del sustrato.}

- Precipitación amorfa o precipitación de cristales de fosfato muy finos (amorfos).

- Cristalización y crecimiento de los cristales.

- $\quad$ Degradación de la capa de cristales.

- Reorganización cristalina.

La primera etapa consiste en la corrosión del sustrato por parte de la solución ácida fosfatizante. El hierro del acero se oxida a $\mathrm{Fe}^{+2}$, a su vez el ion hidronio, $H^{+}$es reducido a hidrógeno molecular promoviendo una elevación del pH interfacial, sustrato-solución. Esta condición provoca la siguiente etapa del fosfatizado, la precipitación de fosfatos muy finos en la superficie del acero. Cabe mencionar que la segunda etapa no se vuelve a presentar durante el proceso, es la única etapa que se presenta solo una vez en el fosfatizado. La Figura 2, esquematiza el ciclo de fosfatizado de manganeso en acero. La etapa de crecimiento de los cristales consiste en la precipitación de cristales de fosfato ricos en manganeso [17]. 


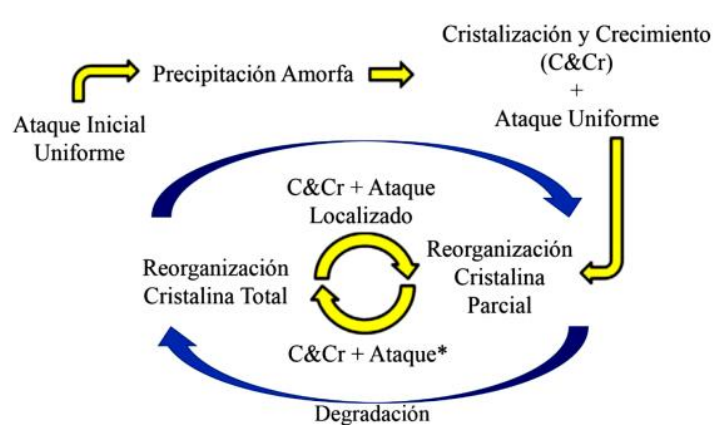

Figura 2 Esquematización del ciclo de fosfatizado con manganeso en aceros. El ataque sobre el acero es uniforme solo en el primer ciclo, habiéndose formado la capa interna, el ataque es localizado [17]

La degradación cristalina tiene lugar durante todo el proceso de fosfatizado y se puede apreciar a través de la formación de lodos en el baño y la apariencia degradada de los cristales en el recubrimiento. La degradación de la capa de cristales se da debido a un fenómeno de acidificación localizada en el interior de los cristales. Defectos en los cristales tales como grietas, hoyos, picaduras etc. favorecen este fenómeno. Durante la acidificación, la superficie del cristal y el área externa de la fosa (picadura, hoyo, grieta, pozo) tienen fácil acceso al oxígeno mientras que la zona interna de la fosa no lo tiene. Por lo tanto, esta zona se convierte en una zona anódica que permite la producción de protones debido a la hidrólisis continua que se produce dentro de estos defectos. En las inmediaciones del pozo, es muy favorable la hidrólisis $\left(\mathrm{H}_{2} \mathrm{O} \rightleftharpoons 2 \mathrm{H}^{+}+1 / 2 \mathrm{O}_{2}+2 e\right)$ debido a la presencia de $\mathrm{NO}_{3}^{-}$y $\mathrm{H}_{2} \mathrm{PO}_{4}^{-}$, la alta relación de área de cátodo/ánodo, la alta temperatura cuando el proceso se lleve a cabo a temperaturas elevadas [17].

El proceso de acidificación modifica la composición química de los cristales de hureaulita ricos en Mn, mediante separación por arrastre de manganeso, un proceso análogo a la lixiviación dando como resultado la disminución del tamaño del cristal. Este proceso inicia hasta que el $\mathrm{pH}$ local cae por debajo de 2.6 ya que la hureaulita rica en $\mathrm{Mn}$ se disuelve a $\mathrm{pH}$ menores de 2.6. Como se puede ver en la Figura 3, por debajo de este $\mathrm{pH}$ sólo queda hureaulita rica en $F e$. Todo esto conlleva a una reducción del peso del recubrimiento debido a que el contenido de $\mathrm{Fe}$ en el cristal es inferior al de manganeso.
Cuando la composición de la hureaulita se modifica, una reorganización cristalina tiene lugar y conduce un cambio de morfología de cristales prismáticos a una morfología de película, es decir, los cristales prismáticos de hureaulita ricos en $M n$ sobre la superficie del revestimiento se sustituyen por una película de hureaulita rica en $\mathrm{Fe}$ (capa interna) [17].

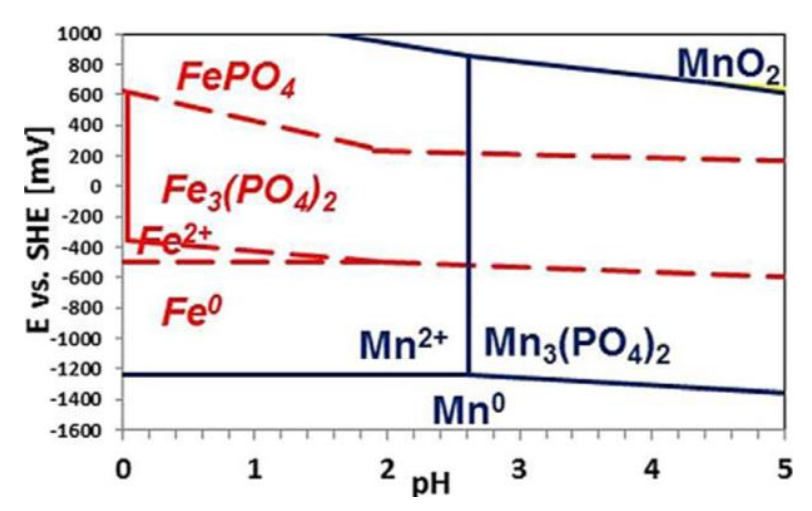

Figura 3 Diagrama de Pourbaix a $90^{\circ} \mathrm{C}$ para el $\mathrm{Mn}-$ $\mathrm{H}_{3} \mathrm{PO}_{4}$ (línea sólida) y $\mathrm{Fe}-\mathrm{H}_{3} \mathrm{PO}_{4}$ (línea punteada) [17]

El proceso para el cálculo del mecanismo fenomenológico del fosfatizado seguido en este trabajo se basó en ir pesando la muestra y verificar cómo va cambiando el OCP. Se observó el incremento de la masa en la muestra y se verificó el potencial de circuito abierto. Mediante lo anterior se identificó en qué etapa iba el fosfattizado, cruzando los resultados con microscopía electrónica.

\section{Metodología}

Los sustratos empleados para los experimentos de fosfatizado con manganeso por inmersión son láminas de acero formable de bajo carbono. Los sustratos empleados para la realización de las pruebas experimentales fueron una lámina recocida de acero formable IF (libre de intersticiales) etiquetada como $\mathrm{S}$ (composición del carbono de $0.009 \%$ en peso) y dos láminas de acero de bajo carbono etiquetadas como $\mathrm{C}$ ( $0.058 \%$ en peso) y $\mathrm{D}(0.038 \%$ en peso $)$.

Los aceros $\mathrm{C}$ y $\mathrm{D}$ tienen un bajo contenido de fósforo y niobio con respecto al acero $\mathrm{S}$. Con lo que respecta al tamaño de grano en el acero $\mathrm{S}$ tiene un tamaño de grano más uniforme y más pequeño, contrastando con el acero $\mathrm{C}$ que es el que exhibe un tamaño de grano mayor y menor uniformidad en los tamaños. 
Para observar el comportamiento del recubrimiento a diferentes acabados superficiales de los aceros, las muestras se desbastaron por uno de los lados con lijas de carburo de silicio (SiC) de números 320 y 1200 en presencia de agua y enjuagadas con agua desionizada. Los lados se etiquetaron como A a los lados desbastados y los que no como lados E.

Los reactivos empleados fueron de grado analítico: dióxido de manganeso (99.99\%, Aldrich Chemistry), ácido fosfórico $(85.8 \%$, J.T. Baker), ácido nítrico (65.7\%, J.T. Baker), hidróxido de sodio (98.3\%, J.T Baker), además se usó agua desionizada (18.2 $\mathrm{M} \Omega-\mathrm{cm})$ para preparar las soluciones, dicha agua fue obtenida con el desionizador Barnstead modelo D7031.

El fosfatizado se realizó empleando el método por inmersión. Las etapas del fosfatizado son ocho operaciones, sin embargo, en dependencia de las condiciones superficiales del sustrato (metal base), algunas se pueden omitir o agregar al sistema. Una secuencia típica se ilustra en la Figura 4 [3]. Un proceso típico de fosfatizado mediante un proceso con limpiadores separados que está basado en un fosfato de sodio o de amonio con acelerantes y producen recubrimientos en un rango de 0,3 a $0,6 \mathrm{~g} / \mathrm{m}^{2}$. La secuencia característica de operación puede ser la siguiente:

- Desengrase alcalino: 1 o 2 minutos por spray, o 2 a 4 minutos por inmersión.

- Decapado: se emplea por lo general mediante una solución de ácido fosfórico al $20 \%$ y a una temperatura entre 60 y 70 ${ }^{\circ} \mathrm{C}$ produciendo un desoxidado en tiempos razonables.

- Enjuague: 30 segundos por spray o 1 minuto por inmersión.

- Activación: Se lleva a cabo con la adición de agentes activadores, lo cual permite incrementar el número de lugares activos de la superficie del metal, donde se forma el cristal de fosfato.

- $\quad$ Fosfatizado: 1 a 2 minutos por spray o 2 a 4 minutos por inmersión.

- Enjuague: 30 segundos por spray o 1 minuto por inmersión.
- $\quad$ Sellado: 30 segundos por spray o 1 minuto por inmersión.

- Secado: se emplea para eliminar cualquier residuo de agua del lavado, en algunos casos en el secado se origina una película con una estructura tridimensional causada en algunos casos por la macromolécula de un polímero.

Las temperaturas operativas pueden variar entre $30^{\circ} \mathrm{C}$ y $75^{\circ} \mathrm{C}$ dependiendo de las concentraciones. $\mathrm{El}$ enjuague final con un sellador orgánico o crómico es un aporte importante del proceso. Este puede ser seguido por un enjuague final con agua desionizada.

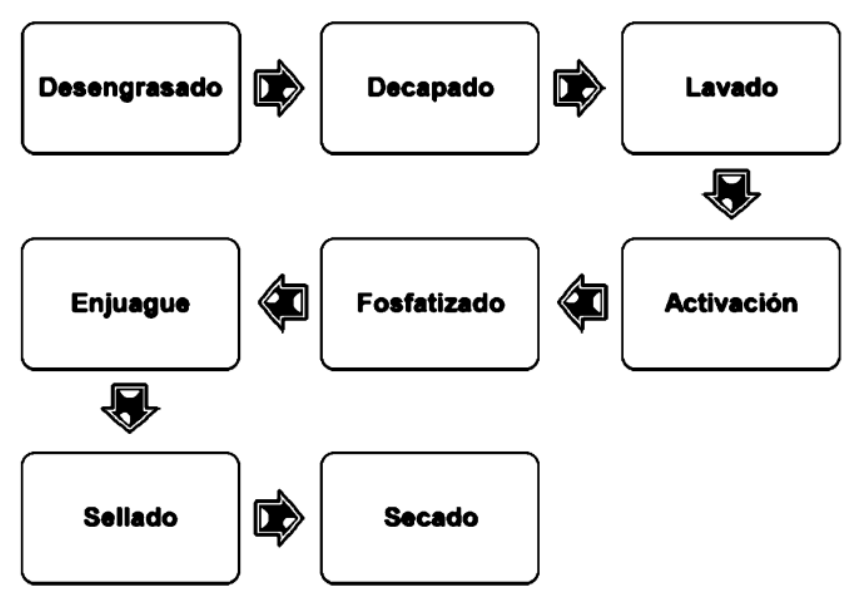

Figura 4 Secuencia de fosfatizado mediante el método de inmersión [3]

Los aceros fueron sometidos a un proceso de fosfatizado, con la finalidad de monitorear la cinética de formación del recubrimiento de fosfato de manganeso y evaluar los cambios microestructurales a distintos tiempos de procesamiento mediante microscopía electrónica de barrido. Para este proceso se cortaron 10 piezas de las láminas de cada acero con dimensiones de $1.8 \mathrm{~cm}$ por 0.8 $\mathrm{cm}$. Las condiciones de trabajo a las cuales se fosfatiza son una temperatura de $90{ }^{\circ} \mathrm{C}$, con tiempos de inmersión de 1, 5, 10,15, 20, 25, 30, 35,40 y 45 minutos para cada acero por separado. Cabe mencionar que todas las piezas se sumergieron al mismo tiempo, no pieza por pieza, se saca posteriormente una muestra de la solución a los tiempos ya mencionados. Dado que la temperatura es elevada, el agua de la solución tiende a evaporarse, por lo que se agregaba agua desionizada a la solución para mantener un volumen constante de solución. 
Todo este proceso se realizó en un vaso de precipitado de $150 \mathrm{ml}$ con $100 \mathrm{ml}$ de solución fosfatizante, en una parrilla de calentamiento sin agitación y usando un termómetro para medir la temperatura del baño.

La técnica de OCP se utilizó para monitorear la cinética de fosfatizado, esta técnica se utilizó bajo las mismas condiciones de temperatura y volumen constante descritas en el apartado anterior. El tiempo de la prueba fue de 45 minutos. Para cada acero se creó un electrodo montado en resina epóxica con la finalidad de aislar el acero y que solo estuviera en contacto un lado de la muestra, dicha muestra tiene dimensiones de $1 \mathrm{~cm}$ por $1 \mathrm{~cm}$, a su vez la laminilla de acero fue soldada a un alambre de cobre para poder transmitir la señal eléctrica, que después se recubrió con silicón en frío para aislarlo. Este electrodo de trabajo (WE), se montó en una celda electroquímica de 3 electrodos, construida con un vaso de precipitado de $150 \mathrm{ml}$ instrumentado con un termómetro, un electrodo de referencia (REF) de $\mathrm{Ag} / \mathrm{AgCl}$ de la marca Thermo Scientific Orion acoplado a un capilar de Luggin, una barra de grafito como contra-electrodo (CE). La celda electroquímica se colocó en una parrilla de calentamiento para regular la temperatura del baño sin agitación. En la Figura 5 se muestra el montaje de la celda.

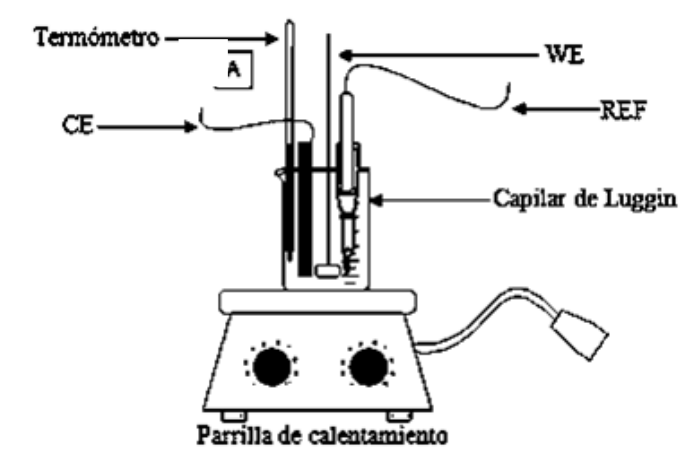

A)

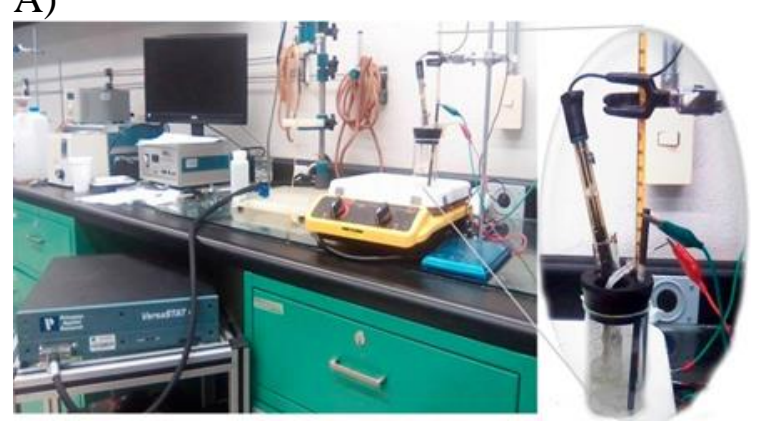

B)

Figura 5 Celda electroquímica de 3 electrodos. A) Esquemática, B) Celda empleada

\section{Resultados}

\section{Evaluación de la cinética de fosfatizado mediante la técnica electroquímica de $\mathrm{OCP}$}

La cinética del proceso de fosfatizado se monitoreó censando el potencial de circuito abierto de cada acero en la solución fosfatizante.

La variación del potencial entre cada prueba de cada acero al tiempo cero no varía demasiado, no difieren en más de -1.22 centésimas de voltio, la cual representa la máxima variación encontrada, en este caso se refiere al acero $\mathrm{C}$, mientras que para los otros dos aceros la variación todavía es menor.

Para los tres aceros existen cinco cambios principales en el potencial. El primer cambio sucede a tiempos cortos, el potencial de circuito abierto se hace más negativo.

El segundo cambio sucede a partir de ese valor mínimo, el potencial aumenta conforme transcurre el tiempo, llegando hasta un potencial máximo relativo, donde el potencial llega a estabilizarse, generando una meseta, la generación de la meseta es el tercer cambio en el potencial, este cambio es más evidente en el acero $\mathrm{S}$.

Después de que el potencial llega a estabilizarse en ese máximo relativo, el potencial aumenta nuevamente (cuarto cambio en el potencial) llegando a un valor de potencial máximo, después del cual se produce un nuevo y último cambio de pendiente, a este nuevo cambio de pendiente se le nombra, en este trabajo, potencial estabilizado, el aumento del potencial en esta etapa es muy lento, o prácticamente despreciable (Figura 6).
GÓMEZ-MACÍAS, Josué, CONEJO-FLORES, Ricardo, GARCÍA-GONZÁLEZ, Juan Manuel y FUENTESACEITUNO, Juan Carlos. Efecto del tipo de acero y su acabado superficial sobre la cinética de fosfatizado con manganeso. Revista. Revista de Ingeniería Innovativa. 2020 


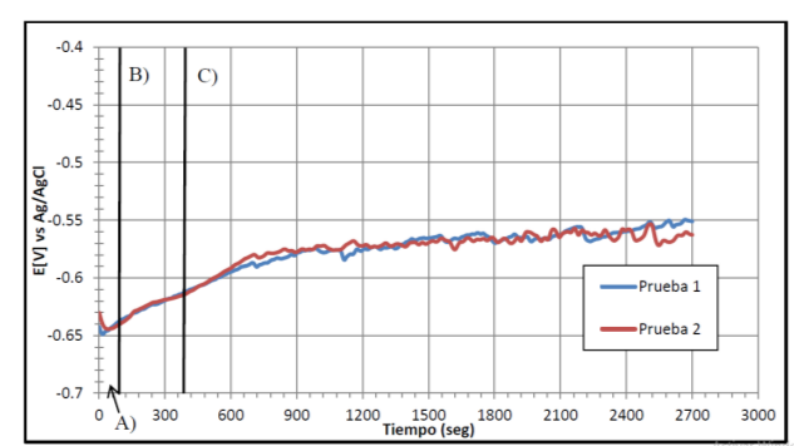

Acero $\mathrm{C}$

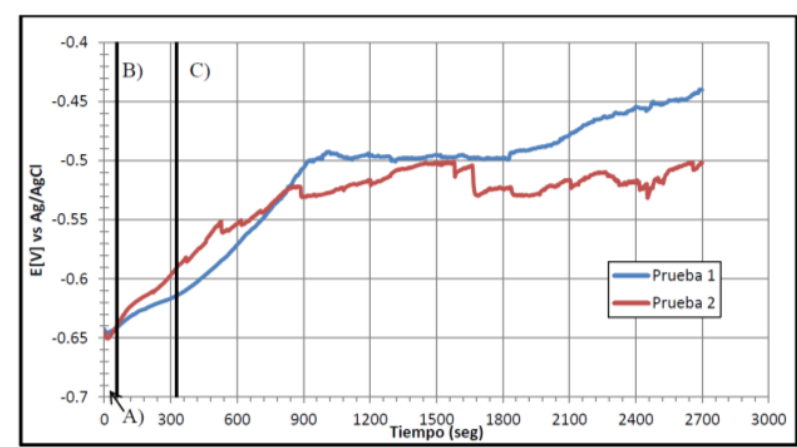

Acero D

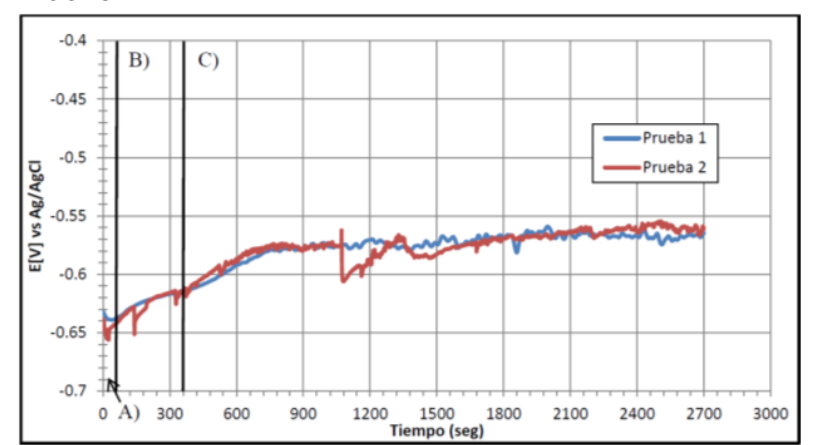

Acero $\mathrm{S}$

Figura 6 OCP de los aceros C, D y S del lado desbastado en solución fosfatizante II a $90{ }^{\circ} \mathrm{C}$

A partir del análisis de las curvas de OCP mostradas en las Figuras 6, se deduce: durante la etapa (A), existe una disminución del potencial, y es la etapa inicial de corrosión del sustrato. Este fenómeno de corrosión se observó en las pruebas efectuadas con los tres aceros, y es consistente con lo reportado en la literatura, donde el acero sufre una corrosión previa al inicio de la deposición de los cristales de fosfato de manganeso. Por otro lado, el comportamiento de las curvas de OCP en la Figura 6, es el característico de la formación del recubrimiento de fosfato de manganeso. La segunda etapa (B) de la técnica de OCP se aprecia en el incremento rápido del potencial, llegando a un cambio de pendiente o generación de meseta que se relaciona con la formación del recubrimiento, en algunos aceros es más apreciable la formación de dicha meseta. Para el acero S (Figura 6), la formación de la meseta es más perceptible que para los otros dos aceros.
Durante esta etapa, para la réplica del acero $\mathrm{S}$ se pueden ver dos decaimientos significativos del potencial, esto puede ser atribuible a la acumulación de burbujas de hidrógeno $\left(\mathrm{H}_{2}\right)$ en la superficie del sustrato. La formación de la meseta en el acero D (Figura 6) no es muy apreciable ya que el crecimiento del recubrimiento en esa etapa es muy rápido. En la tercera etapa el incremento en OCP ocurre hasta un valor máximo ( $\left.\mathrm{E}_{\mathrm{máx}}\right)$ para la mayoría de los aceros, este máximo indica el tiempo requerido para que la superficie del sustrato haya sido recubierta completamente con los cristales de fosfato de manganeso [2,10]. Dichos tiempos entre cada acero son muy semejantes.

El acero que alcanzó el potencial máximo más rápido en la tercera etapa fue el acero $\mathrm{C}$ (Figura 6), lo que indica que fue el que tiene una mayor cinética de fosfatizado, lo que revela que es el acero que se recubre en menor tiempo, a comparación de los otros dos aceros, seguido del acero $\mathrm{S}$ con una diferencia de no más de 55 segundos. Después del potencial máximo, el potencial tiende a estabilizarse, alcanzando un pequeño incremento en su valor, en la mayoría de los aceros. El incremento en el valor del potencial después del potencial máximo se debe a la reorganización y recristalización del recubrimiento, donde el potencial se ve afectado solamente por efecto de los ciclos de degradación parcial o total de los cristales seguidos de una recristalización. Es interesante comentar que, durante la etapa de reorganización cristalina, en donde los cristales de fosfato de manganeso se disuelven y re-precipitan de manera cíclica, el potencial registrado en el material no disminuye hasta su valor original.

\section{Caracterización química y microestructural de los recubrimientos}

Las piezas sometidas al proceso de fosfatizado se analizaron por MEB para evaluar la morfología y la composición química de los recubrimientos con respecto al tiempo de inmersión. En dicho análisis se tomaron fotomicrografías de las placas de acero sumergidas a los diferentes tiempos en el baño fosfatizante. En dichas muestras, se forma un recubrimiento de cristales de fosfato de manganeso sobre la superficie. 
Los cristales formados en ambos lados de los aceros (i.e., desbastado y lado de llegada) tienen una composición rica en fosfato de manganeso con poca concentración de hierro. El porcentaje en peso de contenido de manganeso en los cristales va hasta un $40 \%$ en su estructura, aunque se encontraron cristales con un contenido mayor de manganeso. Como se puede observar en la Figura 7, el recubrimiento está conformado por fosfatos de manganeso en su mayoría, existiendo poca cantidad de hierro en los cristales.
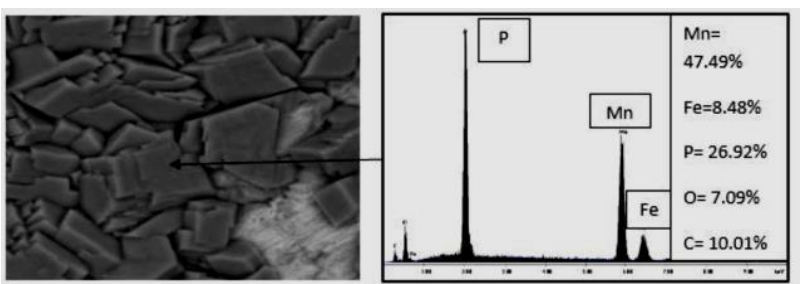

Figura 7 Recubrimiento del acero "S" por el lado desbastado a 15 minutos de inmersión en el baño fosfatizante

La forma que adoptan los cristales es prismática con formas muy irregulares. Como puede verse en la Figura 8, para el caso del acero C fosfatizado por lado desbastado. Esto es de esperarse ya que la hureaulita de fosfato de manganeso forma cristales prismáticos y no películas como es el caso de la hureaulita de hierro [2], estos prismas tienen una tendencia a formar un hexágono en al menos uno de los lados del prisma, este hexágono puede ser regular o irregular, alargado en sentido de un par de sus vértices opuestos o alargado en el sentido de un par de sus lados opuestos o alguna combinación de éstas.
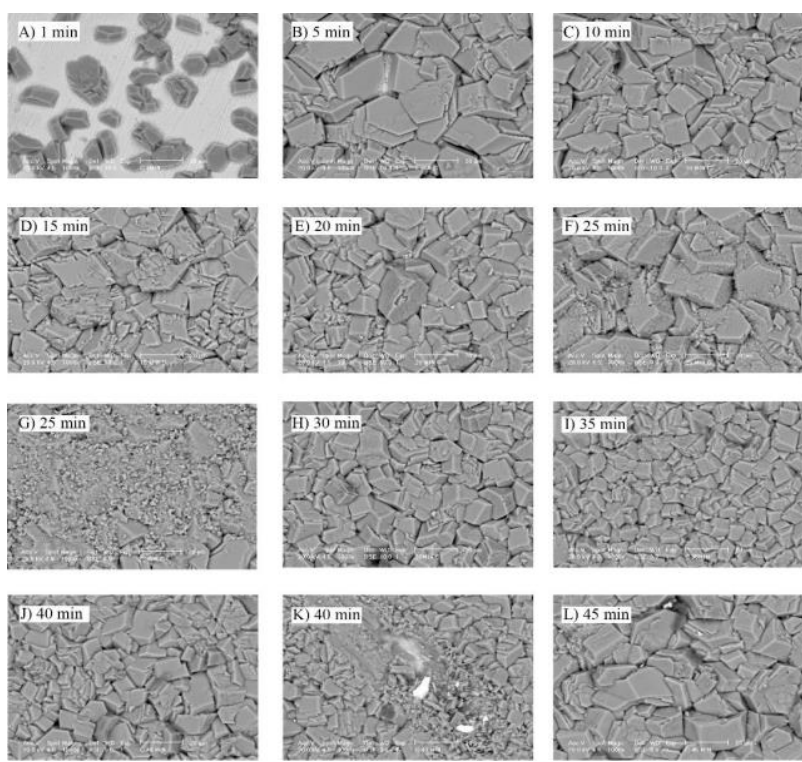

Figura 8 Evolución del recubrimiento en el acero C por el lado desbastado
Durante el crecimiento del recubrimiento se observó otro factor que promueve la irregularidad de la forma de los cristales: los defectos presentes en ellos, como son la formación de láminas o capas incompletas, maclas y picaduras en los cristales. Las láminas o capas incompletas delimitan el crecimiento del cristal, provocan que no siga creciendo el cristal en cierta dirección, esto a su vez crea huecos.

Las maclas son un crecimiento irregular en alguna estructura debido al acomodo y/o orientación de las moléculas en su estructura [1], esto ocasiona un cambio de la dirección de crecimiento del cristal. Las picaduras se presentan durante todo el proceso de fosfatizado, siendo más evidentes en el acero D por el lado desbastado (ver Figura 9, las picaduras están indicadas con flechas), estas picaduras son ocasionadas parcialmente debido a la presencia de hierro en la estructura del cristal que crea vacancias en el cristal, por otro lado, la acidificación del cristal descrito anteriormente [2]. Todo esto en su conjunto contribuye a la forma irregular de los cristales. A pesar de que los cristales siguen la tendencia de adoptar formas irregulares, en algunos casos, en ciertas regiones, la forma de los cristales cambia.
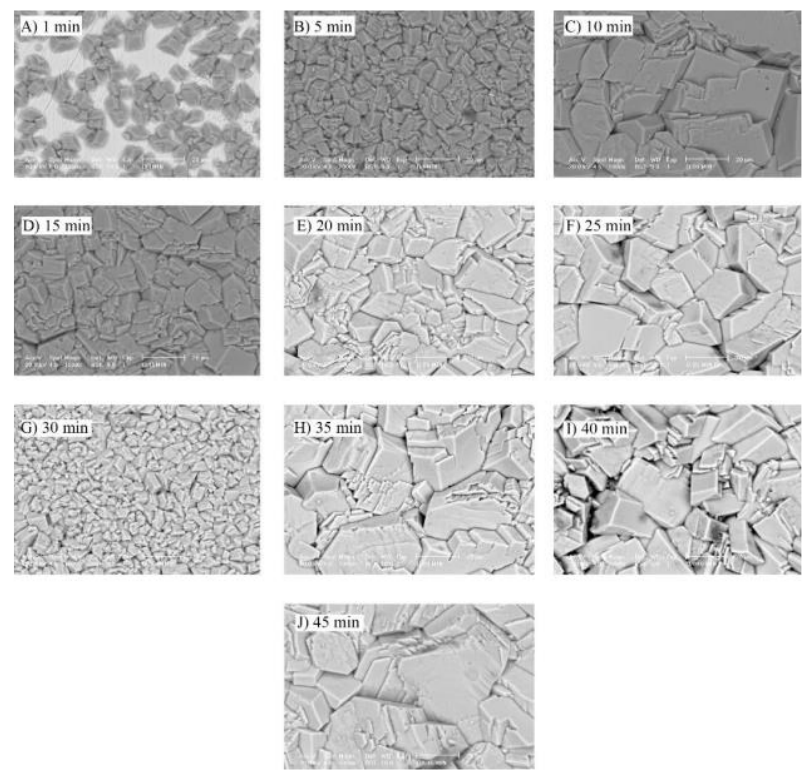

Figura 9 Evolución del recubrimiento en el acero D por el lado desbastado. Las flechas indican la localización de las picaduras en los cristales

Para el acero C lado desbastado (Figura 8), hubo cambio en la forma de los cristales con respecto a la forma típica. A los 40 minutos en una región de la muestra se generaron cristales con forma acicular en las inmediaciones de una zona aparentemente no recubierta Figura $8 \mathrm{~K}$ ).

GÓMEZ-MACÍAS, Josué, CONEJO-FLORES, Ricardo, GARCÍA-GONZÁLEZ, Juan Manuel y FUENTESACEITUNO, Juan Carlos. Efecto del tipo de acero y su acabado superficial sobre la cinética de fosfatizado con manganeso. Revista. Revista de Ingeniería Innovativa. 2020 
Esta zona está recubierta en parte por fosfatos de manganeso y fosfatos de hierro el cual pertenece a la capa interna que se genera en el recubrimiento del acero. En el lado de llegada de este mismo acero no se presentó un cambio en la forma de los cristales como se puede observar en la Figura 10. Los resultados de la Figura 8 y 10, revelan que la superficie tiene un efecto importante en la morfología de los cristales a tiempos largos (aproximadamente de $40 \mathrm{~min}$ ), lo que indica que la rugosidad de la superficie es fundamental para acelerar la cinética de fosfatizado y por otro lado para determinar el tipo y tamaño de cristal requerido.

El acero D presentó cambios en la forma típica de los cristales en ambos lados i.e., desbastado y lado de llegada (ver Figuras 9 y 11). Por el lado desbastado se presentó un comportamiento granular al minuto 30, Figura 9 G). Por el lado de llegada del acero "D" (Figura 11) al minuto de estar sumergida la muestra en el baño fosfatizante, los cristales tienen dos comportamientos, uno con forma típica, Figura 11A), y el otro granular, como se puede ver en la imagen 11B). En la muestra se tienen estas dos formas en diferentes zonas.
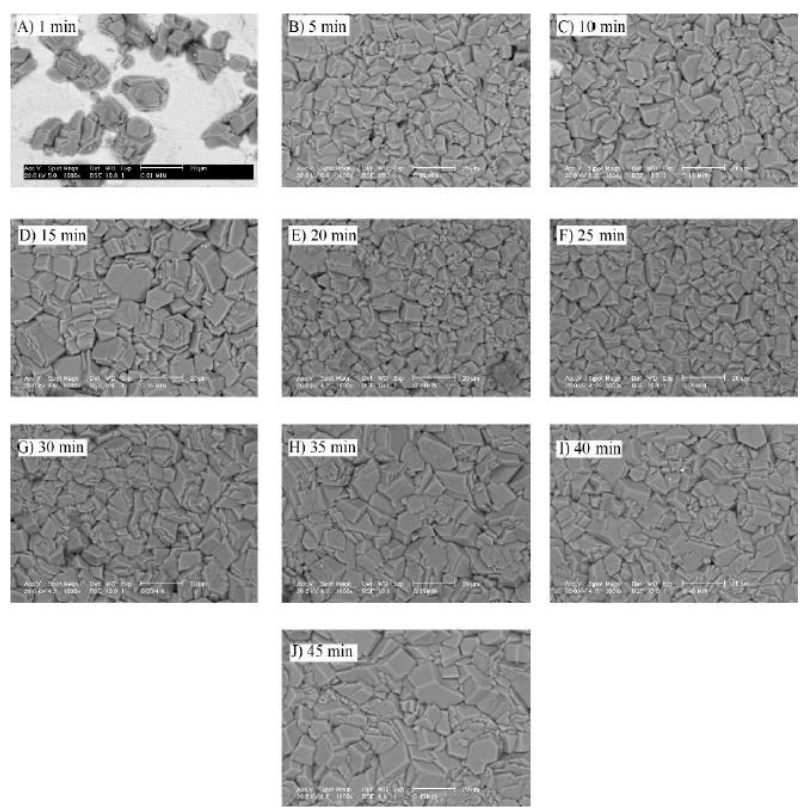

Figura 10 Evolución del recubrimiento en el acero C por el lado de llegada
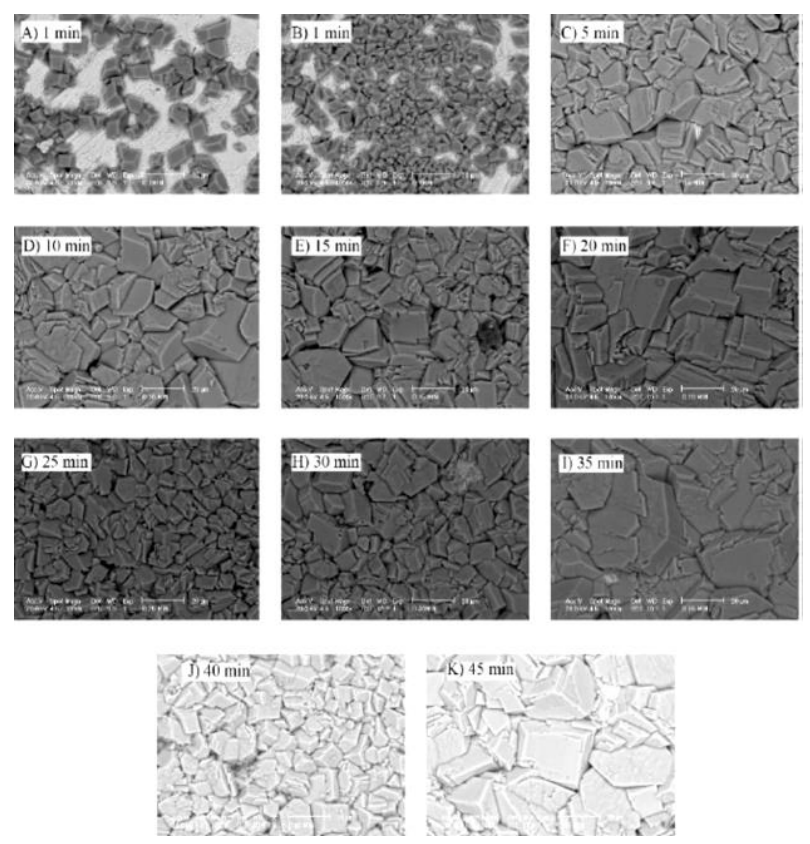

Figura 11 Evolución del recubrimiento en el acero D por el lado de llegada

En el acero S lado desbastado no se presentó un cambio total en la forma de los cristales durante el fosfatizado (Figura $12 \mathrm{~F}$ ), se puede ver la aparición de muy pocos cristales con forma de aguja y en la Figura $12 \mathrm{H}$ ), se presentó una formación parcial de cristales granulares.

Por el lado de llegada del acero $\mathrm{S}$ hubo tres cambios en la forma del cristal, primero los cristales crecían con la forma típica: irregular prismática, al minuto 25 de estar en el baño fosfatizante, se presentó un crecimiento de cristales en forma de gránulos, Figura 13 F), con un tamaño mucho menor, estos cristales granulares no tienden a coalescer unos con otros, como se observa con el recubrimiento de cristales prismáticos. 

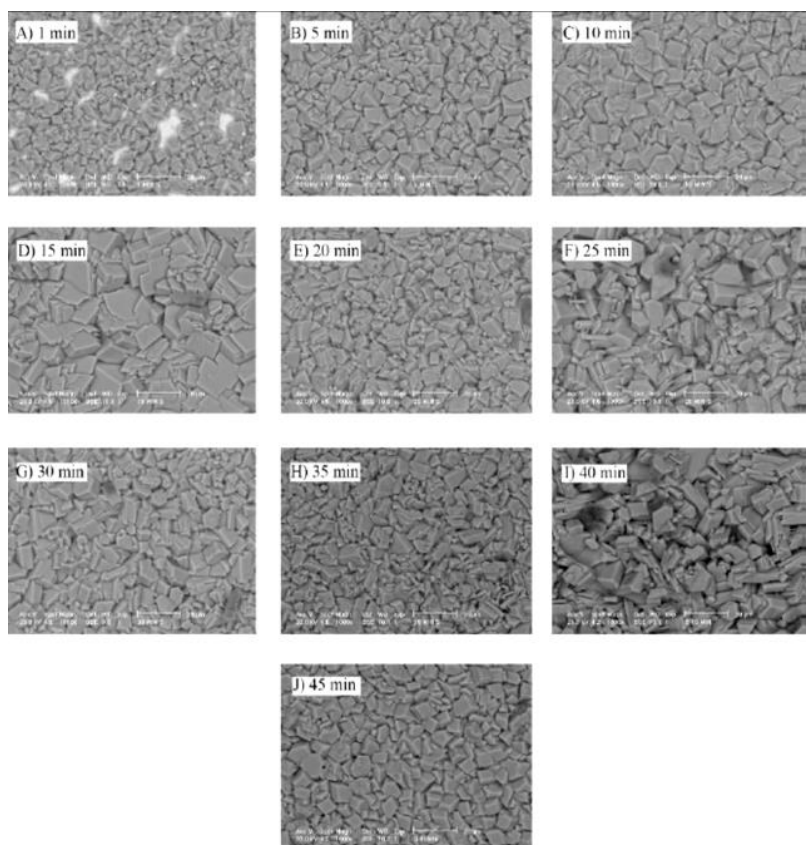

Figura 12 Evolución del recubrimiento del acero S del lado desbastado

El segundo cambio de forma fue al mismo tiempo de inmersión en el baño fosfatizante, pero en una región diferente de la muestra de acero. Dicho cambio tiene forma de agujas, Figura $13 \mathrm{G}$ ), prismas alargados y muy delgados, con apenas unas cuantas micras de espesor. El tercer cambio fue al minuto 30 donde las dos formas antes mencionadas desaparecieron, regresando a su forma típica de prismas irregulares.
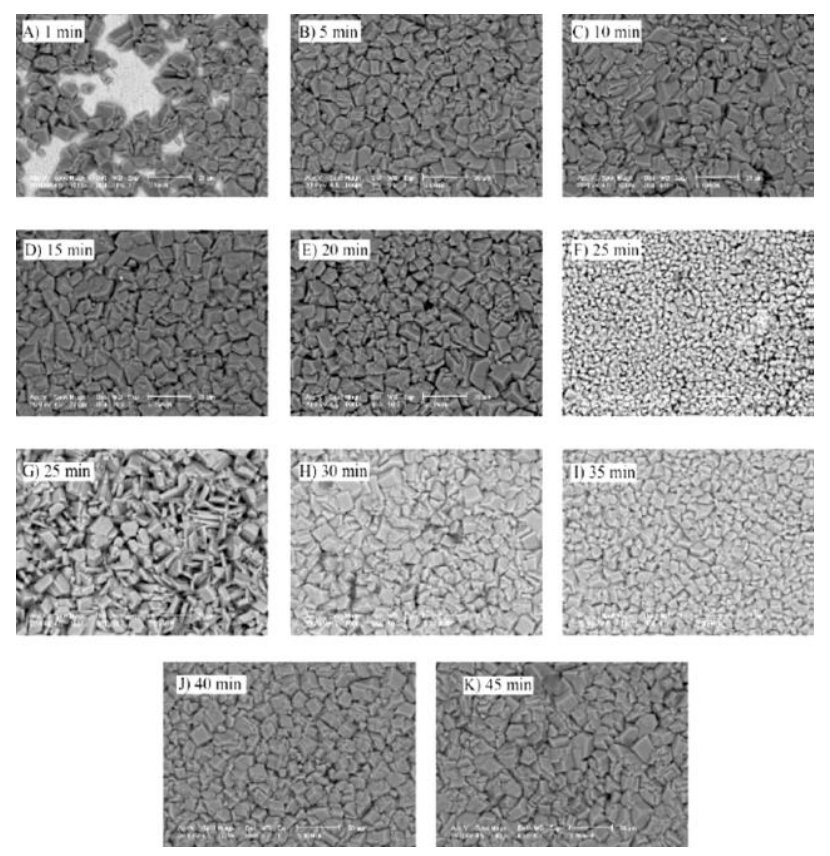

Figura 13 Evolución del recubrimiento en el acero S por el lado de llegada
Los resultados de la Figura 12 y 13 revelan que en efecto la condición superficial del acero (i.e., rugosidad) tiene un efecto importante en la cinética de fosfatizado, de hecho, si se observa al minuto 1 en la Figura 12 y 13, la fracción cubierta del acero por los cristales de fosfato de manganeso es mayor en el acero que fue desbastado que en el fosfatizado sin ningún tratamiento superficial (es decir lado de llegada). Más aún este resultado coincide con lo mostrado en las Figuras 8 y 10 para el acero $\mathrm{C}$ desbastado $\mathrm{y}$ sin tratamiento, respectivamente.

\section{Formación de lodo}

Durante el proceso de fosfatizado se generó lodo. La presencia de este lodo fue visible durante el proceso en la solución fosfatizante. Conforme pasaba el tiempo, la solución fosfatizante, que inicialmente tenía una apariencia incolora, se empezó a opacar, apareciendo partículas de color blanco o de alguna tonalidad de amarillo en la solución. La cantidad de estos lodos fue diferente para cada acero, así como, el tiempo de aparición de este en la solución, para el acero $\mathrm{C}$, apareció a los 2 minutos de estar inmersas en el baño fosfatizante la solución perdió totalmente su cristalinidad, de igual forma para el acero $\mathrm{D}$, a los 3 minutos la solución se turbio y por último para el acero $\mathrm{S}$, a los 8 minutos de fosfatizado, la solución empezó a perder su cristalinidad, señal inequívoca de la formación de lodo.

La generación del lodo, proviene de la desintegración de los cristales, de hecho, un ejemplo de la disolución de los cristales debido a la degradación y reorganización cristalina se puede ver en la Figura $8 \mathrm{G}$ ), esta imagen sitúa al recubrimiento a un tiempo de 25 minutos en el baño fosfatizante.

Los cristales prismáticos de mayor tamaño se están deshaciendo, el producto de la desintegración son cristales de menor tamaño. Una parte de estos cristales de menor tamaño formaran parte del lodo en la solución fosfatizante y otra se recristalizará en el recubrimiento del acero formando nuevos cristales o adhiriéndose a cristales no disueltos. 


\section{Características de los cristales de fosfato de manganeso y su posible relación con la corrosión de los aceros}

A lo largo del proceso de fosfatizado la cantidad de cristales presentes en la superficie de los aceros va cambiando. Como es lógico, al minuto 1 se tiene una cantidad de cristales baja, menos para el acero S (Figuras 12 A) y $13 \mathrm{~A}$ )) donde la densidad cristalina es alta. Después de los 5 minutos de fosfatizado, ya no se puede apreciar con facilidad la superficie del acero. La densidad cristalina para los tres aceros aumenta y disminuye conforme pasa el tiempo, es decir, cambia de manera cíclica.

Con base en las observaciones realizadas en las superficies fosfatizadas de los diferentes aceros se puede decir que en algunos aceros la corrosión fue más homogénea en toda la superficie que en otros, haciendo que en toda o en la mayor parte de la superficie de las muestras se oxidara el hierro (regiones anódicas) y se redujera el hidrógeno (regiones catódicas). Al tener mayor reducción del protón, aumenta el pH interfacial sustrato-solución, favoreciendo la precipitación del fosfato ocasionando una densidad cristalina mucho mayor, pudiendo suponer que la topografía de la superficie fue la causante de tener diferencias al momento de ocurrir el ataque químico. Es decir que, al tener superficies más rugosas, aumenta el área superficial del sustrato y esto a su vez la cantidad de micro-ánodos y micro-cátodos que son los causantes de la precipitación de los cristales de hureaulita ricos en manganeso.

En todas las superficies a tiempos cortos (un minuto de inmersión en el baño fosfatizante) se observó que la densidad cristalina del recubrimiento es diferente. Lo que controla el crecimiento de los cristales es la corrosión del acero, por lo que se puede inferir que en las muestras con densidades cristalinas bajas la corrosión no es tan homogénea en la superficie como en las que hay mayor cantidad de cristales, esto provoca que se tengan áreas muy poco recubiertas a tiempos cortos, además se ve que los cristales que crecen bajo este tipo de corrosión más localizada son más grandes ya que es más factible que siga creciendo el cristal en donde ya hay un asentamiento que encontrar un sitio para que se precipite un nuevo cristal.

\section{Espacios libres observables}

Al minuto del fosfatizado, por los lados desbastados solo hay presencia de pocos cristales para el acero $\mathrm{C}$, siguiendo en cantidad el acero D. En el caso del acero S, el comportamiento es diferente, ya que para este tiempo la superficie ha sido recubierta casi en su totalidad. Esto no contradice lo que se obtuvo en $\mathrm{OCP}$, donde el acero $\mathrm{C}$ es el que se recubre más pronto, ya que el tener una superficie muy poblada de cristales al inicio del proceso no indica que toda la superficie se recubrió por completo, sino que al inicio del fosfatizado la pasivación del metal es rápida y conforme el tiempo transcurre, ésta se hace un poco más lenta.

Esto se debe a que el ataque inicial en el fosfatizado se realiza preferentemente en las fronteras de grano de los aceros y como el acero $\mathrm{S}$ es el que tiene mayor área de fronteras de grano expuestas en la superficie por tener la mayor densidad de granos en la superficie, se favorece la precipitación de fosfatos amorfos de manganeso/hierro al inicio del proceso.

Además, no se ha cumplido ni un ciclo de fosfatizado para ese tiempo de acuerdo con el análisis de OCP. Un comportamiento semejante por los lados de llegada se presentó con respecto a la densidad cristalina donde el acero $\mathrm{S}$ fue el que tuvo mayor densidad cristalina a este mismo tiempo, seguido del acero D y al final el C.

Para los aceros $\mathrm{C}$ y $\mathrm{S}$ se observó que entre los minutos 10 y 15 la superficie está prácticamente recubierta para ambos lados de la pieza y para el acero D entre los minutos 15 y 20. A tiempos posteriores lo que cambia en los revestimientos es el tamaño de los cristales y la densidad superficial cristalina.

El desarrollo de la película interna sucedió tiempo después de que los diferentes aceros llegaron al potencial máximo. En la Figura 14 se puede ver que se ha formado la capa interna, esto soportado por el análisis químico que siguiere que lo que se formó es hureaulita de hierro. Esta aseveración es para los lados desbastados de las muestras. 
Para los lados de llegada sucedió un comportamiento diferente, la formación de la capa interna sucedió antes de que las muestras llegasen al potencial máximo, cabe mencionar que para los lados de llegada no se les realizaron pruebas de OCP, por lo que no se tiene un valor de potencial máximo para las muestras sin acabado superficial, solo se tiene de referencia el potencial máximo de los lados desbastados. Esto sugiere que para los lados de llegada se alcanza más rápido el potencial máximo en comparación con el lado desbastado.

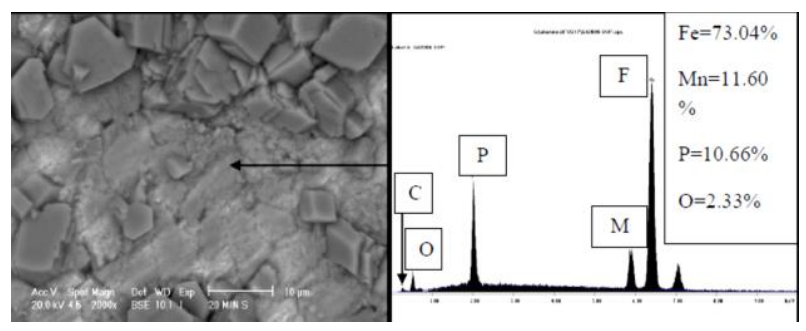

Figura 14 Formación de la capa interna en el acero $\mathrm{S}$ lado desbastado

En la Tabla 1 se muestran los tiempos a los cuales las muestras llegaron a su potencial máximo comparando con el tiempo donde se encontró la capa interna.

\begin{tabular}{|c|c|c|c|}
\hline Acero & $\begin{array}{c}\text { Acabado } \\
\text { Superficial }\end{array}$ & $\begin{array}{c}t_{\text {máx }} \\
{[\mathrm{min}]}\end{array}$ & $\begin{array}{l}\text { tcapa interna } \\
\text { [min] }\end{array}$ \\
\hline \multirow[t]{2}{*}{$\mathrm{C}$} & Desbastado & $\begin{array}{r}11.58- \\
11.92 \\
\end{array}$ & 15 \\
\hline & Llegada & ------------- & 15 \\
\hline \multirow[t]{2}{*}{$\mathrm{D}$} & Desbastado & $\begin{array}{r}14.42- \\
15.62\end{array}$ & 20 \\
\hline & Llegada & ------------- & 10 \\
\hline \multirow[t]{2}{*}{$\mathrm{S}$} & Desbastado & $12.7-12.75$ & 20 \\
\hline & Llegada & -------------- & 10 \\
\hline
\end{tabular}

Tabla 1 Tiempos a los cuales se encontró la capa interna y el potencial máximo para los diferentes aceros, tomando como unidades del tiempo minutos

Se observó que a tiempos menores de donde debe aparecer la capa interna, señalados en la Tabla I, aún no se ha desarrollado la capa interna, sin embargo, existe presencia de precipitados de fosfatos amorfos sobre la superficie expuesta, como se puede observar en la Figura 15 en las zonas no recubiertas por los cristales. Por otro lado, en algunas zonas muy pequeñas de los aceros a tiempos menores de la formación de la capa interna, hay presencia de hureaulita de hierro, no quiere decir que se hubiese desarrollado la capa interna por completo en toda la pieza, simplemente en esas zonas empezó a desarrollarse esta capa.

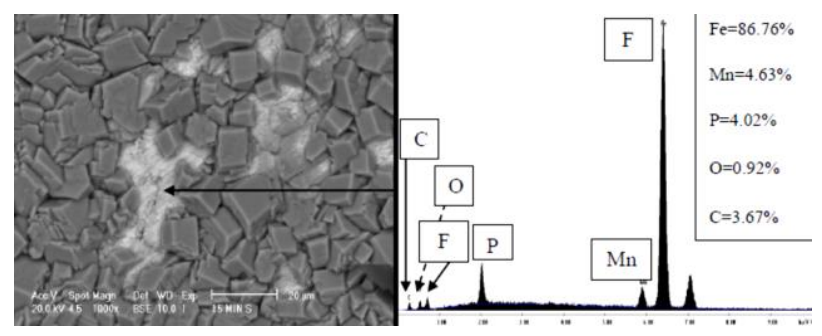

Figura 15 Composición de la superficie del acero $\mathrm{S}$ por el lado desbastado a 15 minutos de fosfatizado

La capa interna está compuesta por hureaulita rica en hierro con poca concentración de manganeso. Esta capa, a diferencia de la capa externa, no forma un recubrimiento prismático, sino una película [2].

\section{Tamaño de los cristales en el recubrimiento de los aceros}

Debido a la irregularidad de la forma de los cristales formados en el revestimiento en los aceros, los cristales se midieron de manera que esas mediciones representaran lo más posible la forma del cristal. Ejemplo de la forma en que se midieron los cristales se muestra en la Figura 16, las mediciones se llevaron a cabo haciendo uso del software Image-Pro® Plus.

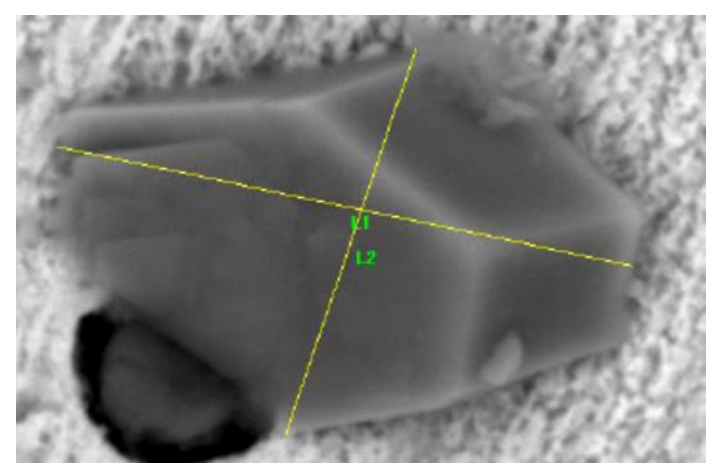

Figura 16 Medición ejemplo de un cristal de fosfato de manganeso en la superficie del acero $\mathrm{C}$ lado desbastado a 1 minuto de fosfatizado

Las mediciones se hicieron para determinar el tamaño promedio de los cristales de la capa externa, más no se realizaron mediciones de espesor, tanto para la capa interna rica en hierro como para la capa externa rica en manganeso. Estas mediciones no arrojan un tamaño del cristal en 3 dimensiones, sino que solamente las medidas del recubrimiento en una vista superior. 
El comportamiento del tamaño de los cristales es cíclico, es decir, el crecimiento de los cristales va en aumento y alcanzado un pico de altura, el tamaño del cristal disminuye llegando hasta un mínimo, en donde se da de nuevo el inicio del crecimiento de los cristales. Cabe mencionar que el comportamiento cíclico es continuo, es decir, el proceso de aumento y disminución del tamaño del cristal ocurre durante todo el proceso de fosfatizado. Aunque el fosfatizado de las muestras de los aceros y las pruebas de OCP fueron a 45 minutos, no quiere decir que el proceso del crecimiento de los cristales sea hasta ese tiempo, de haber prolongado el tiempo de las pruebas, se seguiría presentando el comportamiento cíclico del tamaño de los cristales.

Si bien el crecimiento de los cristales es cíclico, no todos tienen los mismos aumentos o disminuciones con respecto a los tamaños, inclusive el comportamiento cíclico varía de lado a lado de cada acero teniendo picos a diferentes tiempos. En unos aceros el número de ciclos presentes durante el proceso son más que en otros. En el acero D se presentaron el mayor número de ciclos durante el fosfatizado llegando a tener 3 ciclos por el lado desbastado y por el lado de llegada 2 ciclos y uno aparente, ver Figuras 17 y 18 . Este ciclo aparente ocurre a los 5 minutos del fosfatizado en el lado de llegada donde se forma una meseta, a los 15 minutos decae el tamaño de los cristales. En este lapso no se presentó un pico como tal y el decaimiento en el tamaño no fue de gran magnitud.

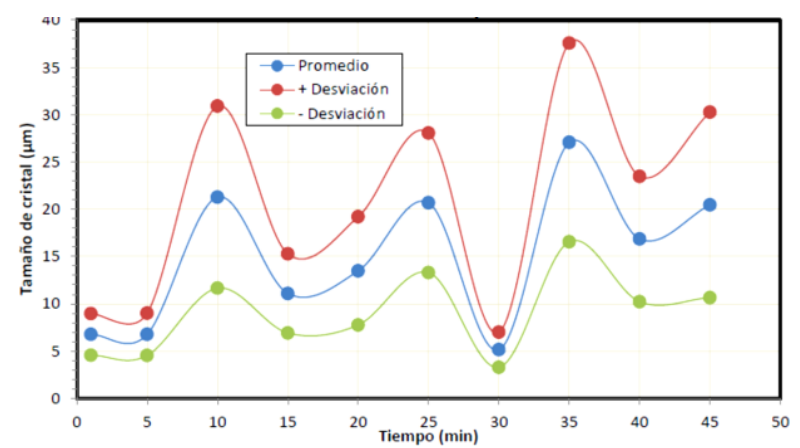

Figura 17 Desarrollo del tamaño de los cristales de hureaulita de manganeso durante el tiempo de fosfatizado a $90{ }^{\circ}$ C. Acero D lado desbastado, Solución 2

Otro comportamiento referente a los ciclos es que para el acero D, Figura 17, los cambios en el tamaño de cristal son más abruptos. Se tiene un crecimiento de los cristales rápido y a su vez una disminución en el tamaño con una rapidez similar.

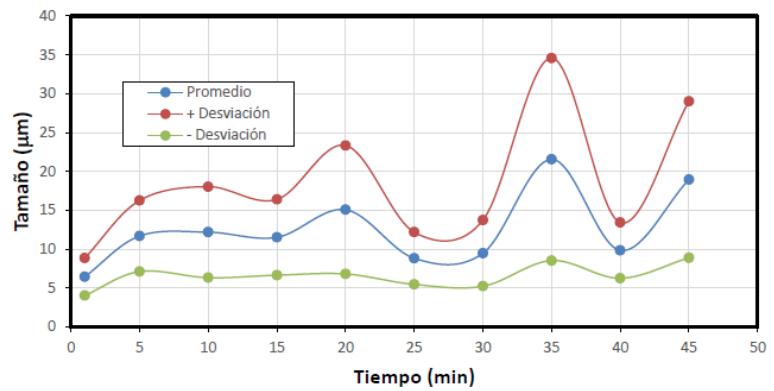

Figura 18 Desarrollo del tamaño de los cristales de hureaulita de manganeso durante el tiempo de fosfatizado a $90{ }^{\circ} \mathrm{C}$. Acero D lado de llegada, Solución 2

Al observar las figuras de los tamaños de los cristales de los aceros C y S los picos generados no son tan delgados, sino anchos como lo es en el acero C (Figura 19 y 20), inclusive podría decirse que son inexistentes como es el caso para el acero $\mathrm{S}$.

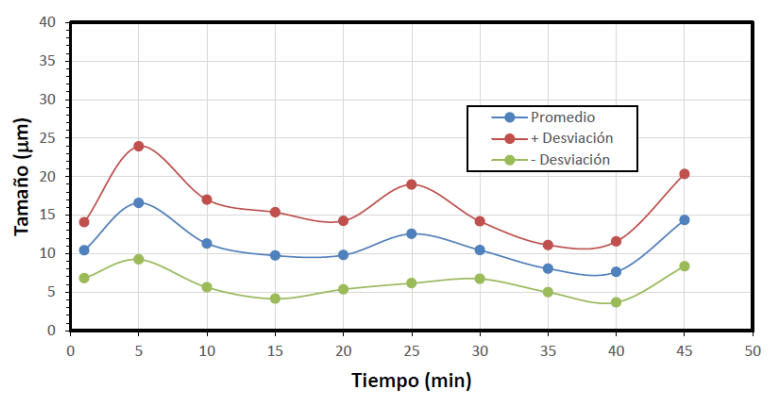

Figura 19 Desarrollo del tamaño de los cristales de hureaulita de manganeso durante el tiempo de fosfatizado a $90{ }^{\circ} \mathrm{C}$. Acero C lado desbastado, Solución 2

Al comparar el lado desbastado del acero $\mathrm{S}$ (Figura 21) con el lado de llegada del mismo acero (Figura 22), el lado de llegada no presenta cambios significativos en el tamaño de los cristales, el mayor cambio presente en este lado del acero $\mathrm{S}$ fue al minuto 25 , a este tiempo se presentó un decaimiento en el tamaño de los cristales, sin embargo, a los siguientes tiempos el tamaño volvió a seguir la misma tendencia de tamaños.

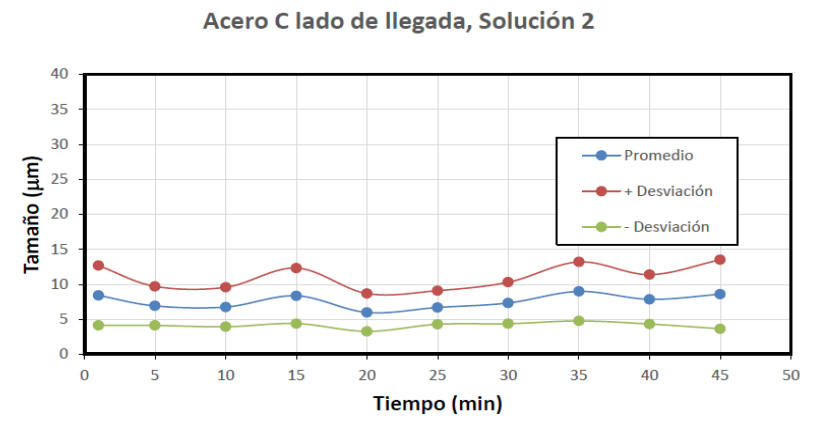

Figura 20 Desarrollo del tamaño de los cristales de hureaulita de manganeso durante el tiempo de fosfatizado a $90{ }^{\circ} \mathrm{C}$. Acero C lado de llegada, Solución 2

GÓMEZ-MACÍAS, Josué, CONEJO-FLORES, Ricardo, GARCÍA-GONZÁLEZ, Juan Manuel y FUENTESACEITUNO, Juan Carlos. Efecto del tipo de acero y su acabado superficial sobre la cinética de fosfatizado con manganeso. Revista. Revista de Ingeniería Innovativa. 2020 


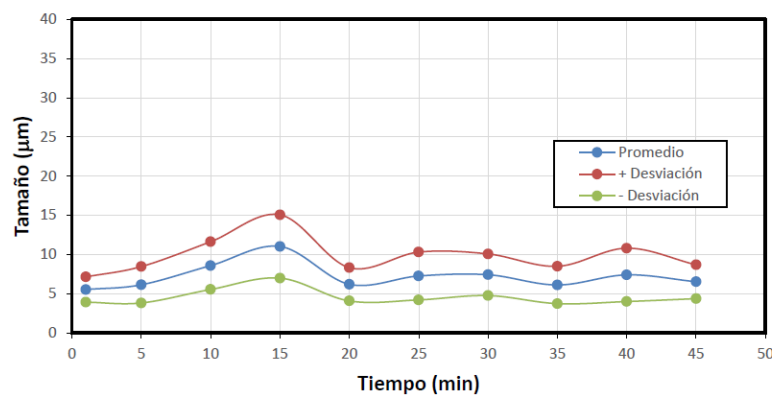

Figura 21 Desarrollo del tamaño de los cristales de hureaulita de manganeso durante el tiempo de fosfatizado a $90{ }^{\circ} \mathrm{C}$. Acero $\mathrm{S}$ desbastado, Solución 1

El presentar picos anchos en las curvas de tamaños de cristal sugiere que el cambio en el tamaño de los cristales para estos aceros es lento. Por lo que los tamaños de los cristales son más homogéneos.

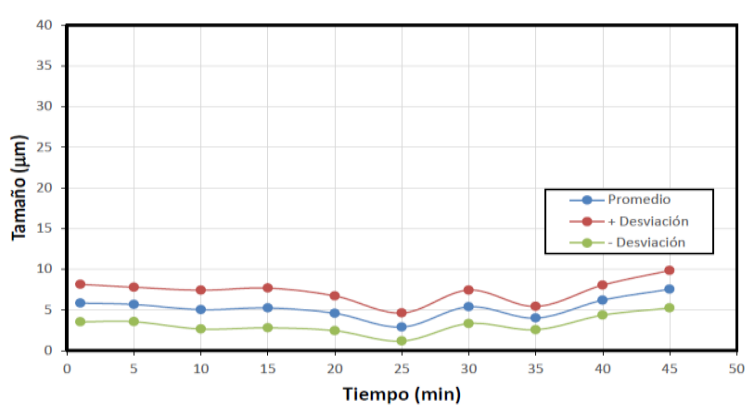

Figura 22 Desarrollo del tamaño de los cristales de hureaulita de manganeso durante el tiempo de fosfatizado a $907^{\circ} \mathrm{C}$. Acero $\mathrm{S}$ lado de llegada, solución 1

El acabado superficial de las muestras afecta directamente al comportamiento cíclico del crecimiento de los cristales, modificando de manera importante el tamaño y heterogeneidad de los cristales, la velocidad de crecimiento y desintegración de los cristales y la facilidad de corroer la superficie ante la solución fosfatizante.

Se observó que en los lados desbastados de las muestras de acero se tuvieron cristales de mayor tamaño en comparación con sus lados de llegada. Con respecto a la heterogeneidad de los cristales, en las gráficas de tamaño de cristal se agregaron dos curvas más, estas curvas corresponden a más/menos una desviación estándar de cada punto de la curva de tamaños promedios de los cristales, la desviación representa el grado de heterogeneidad de los tamaños de los cristales. En los lados desbastados se tiene una mayor desviación con respecto al promedio, lo que indica que a pesar de que se tuvo un tamaño de cristal promedio a cierto tiempo, en toda la muestra se tiene una gran irregularidad de tamaños.
No sólo el acabado superficial afecta la heterogeneidad, sino que también se ve que, al disminuir el tamaño de los cristales durante el proceso, el valor de la desviación estándar en ese punto disminuye, lo que refleja un grado de homogeneidad mayor en los tamaños de los cristales. Cuando los cristales tienden a aumentar de tamaño también lo hace la heterogeneidad ya que la desviación percibida en los momentos de crecimiento de los cristales aumenta con respecto a los valores donde los cristales disminuyen de tamaño. Como puede verse al comparar las Figuras 16, 18 y 20 con las figuras de los aceros por los lados de llegada, Figuras 17, 19 y 21, las curvas de \pm desviación de los lados de llegada están más cercas a la curva de promedio del tamaño de cristal, lo que indica que se tiene más homogeneidad en los cristales formados en los lados de llegada que en los lados desbastados.

En el acero D, la velocidad de crecimiento y desintegración de cristales es mayor que en los otros dos aceros, pero el acabado superficial hace que estos cambios sean aún más abruptos para todos los aceros. Al desbastar la superficie se presentan picos más afilados en las curvas de tamaño que cuando no se desbastan las muestras. El crecimiento de los cristales es más lento y homogéneo en los lados de llegada que en los lados desbastados, aunque el desbastar aumenta el tamaño de los cristales.

La facilidad de corrosión y deposición de cristales aumenta al desbastar la superficie, esta observación solo es posible verse al minuto de fosfatizado, ya que a tiempos posteriores la mayor parte de la superficie es poblada de cristales. Como se puede ver en las Figuras 8 a 13 a tiempo de un minuto, hay mayor número de cristales depositados en las superficies desbastadas que en las no desbastadas.

Independientemente de que acero y lado se trate, el acero D obtuvo los cristales de mayor tamaño. Para el lado desbastado desde tiempos relativamente cortos, este acero ya presentaba cristales de tamaños promedios mayores de 20 $\mu \mathrm{m}$, esto antes de que la superficie se recubriera por completo. El pico máximo se presentó a un tiempo de 35 minutos con un tamaño promedio de cristal de $27 \mu \mathrm{m}$. 
Los cristales de menor tamaño se formaron en el acero $\mathrm{S}$, el tamaño del cristal no excedió las $12 \mu \mathrm{m}$ para el lado desbastado y 8 $\mu \mathrm{m}$ para el lado de llegada. Para el acero $\mathrm{C}$ se tuvieron tamaños de entre 7.6 a $17 \mu \mathrm{m}$ por el lado desbastado y de 5.9 a 9 micras por el lado de llegada.

\section{Conclusiones}

Cuando el acero presenta tamaños de grano pequeños y homogéneos, la velocidad de corrosión del sustrato solamente al inicio del proceso es alta debido a la gran cantidad de límites de grano presentes en la superficie, ya que en estos sitios es donde el ataque se hace preferentemente, lo que indica que la velocidad de fosfatizado sea alta al inicio del proceso.

Un factor determinante que favorece el ataque químico inicial, modificando el tamaño y homogeneidad de los cristales depositados es el acabado superficial que se le realice al acero. El desbastar las superficies de acero a fosfatizar, el ataque químico inicial es homogéneo en toda la superficie teniendo precipitación de cristales de hureaulita ricos en manganeso a tiempos cortos, esto provoca que se obtengan revestimientos más uniformes en toda la superficie, disminuyendo la cantidad de poros en el recubrimiento desde el inicio del proceso. Se igual forma, al desbastar las superficies metálicas, se obtienen cristales de mayor tamaño en comparación a las superficies que no fueron tratadas. Siendo más notorio el aumento del tamaño de los cristales de los revestimientos en el acero D, siguiendo el acero $\mathrm{C} y$ al final el acero S. Agregando al comportamiento presente en las superficies desbastadas, se tiene que, al tratar las superficies de esta manera, el crecimiento y disminución del tamaño de los cristales es mucho más abrupto, es decir, las velocidades de crecimiento y desintegración de los cristales son muy rápidas, ocasionando que los cristales obtenidos en las superficies desbastadas tengan mayor tamaño y mayor heterogeneidad, resultando en revestimientos con porosidad debido a los espacios que dejan los cristales al tener mayor heterogeneidad en el tamaño.

Industrialmente hablando, el tener cristales de menor tamaño al no desbastar las superficies de los sustratos ocasiona que el recubrimiento final tenga menor cantidad de poros.
Otro factor que se ve potenciado al tener cristales de tamaños pequeños es la adherencia y mayor uniformidad de posibles películas aplicadas a las piezas fosfatizadas,

Finalmente, la rugosidad de la superficie del sustrato es un factor importante en el fosfatizado ya que éste dictará si los cristales del recubrimiento tendrán tamaños grandes o de menores dimensiones, impactando en gran medida los tratamientos posteriores que pudieran tener las piezas fosfatizadas de acuerdo a su aplicación. Asimismo, la rugosidad influye en la velocidad de corrosión y en los tiempos a los cuales se presenta la capa interna, por consiguiente, la pasivación total de la superficie del sustrato.

A pesar de que no se realizaron mediciones del potencial de circuito abierto (OCP) para los aceros por el lado de llegada, si se observó que la capa interna aparece antes, a diferencia de las superficies que, si se desbastaron, por lo que se puede inferir que la rugosidad del sustrato acorta el tiempo para que la superficie se pasive.

\section{Referencias}

[1] Callister Jr. W. D. (1995), "Introducción a la ciencia de los materiales", 3ra edición, editorial Reverte S.A., Barcelona, España.

[2] T.S.N. Sankara Narayanan, T. (2005). Surface pretreatment by phosphate conversion coatings - a review. Adv.Mater.Sci. 9, 130-177.

[3] Gruss B. (2010) “Iron phosphating”. Journal Metal finishing, volume 108, issue 1, pages 33-37.

[4] Delgado Aguilar A. (2016). "Estudio Fenomenológico de las etapas críticas del proceso de fosfatizado con $\mathrm{Zn}-\mathrm{Mn}$ de láminas de acero formables", Centro de investigaciones y de Estudios Avanzados del Instituto Politécnico Nacional Unidad Saltillo. 
[5] López León Servando (2008). Distribución del activador de Ti y su efecto en la formación de los recubrimientos de fosfato. Tesis de Maestría. Centro de Investigación y Desarrollo Tecnológico en Electroquímica. Querétaro.

[6] Cruz Vázquez Janet Paola (2010) Recubrimiento de fosfatados sobre acero 304 sensibilizado. Tesis de Maestría CINVESTAV Unidad Altamira. Altamira, Tamaulipas.

[7] Onofre Bustamante E., Olvera A., Barba A., Rodríguez F.J., Carboneras M., Álvarez M.,. García Alonso M.C y. Escudero M.L (2010) Optimización del tratamiento de fosfatado como método de protección frente a la corrosión. Revista de Metalurgia, 46 (4) Julio-Agosto, pp 360-369.

[8] Alvarado García Miguel Ángel (2017) Influencia del potencial, concentración y ph en el fosfatizado electroquímico y su capacidad como inhibidor de la corrosión de un acero AISI SAE 1018. Tesis de Maestría. UAM Azcapotzalco. Ciudad de México.

[9] Calle Bone Lilian Leonor. (2019) Determinación del espesor de la capa de fosfatizado en metales ferrosos en función de la concentración, temperatura y tiempo. Tesis de Licenciatura. Carrera de Ingeniería Química, Facultad de Ingeniería Química, Universidad Central del Ecuador. Quito, Ecuador.

[10]Romero Bonilla Hugo, Vera Aravena Rosa, Redrovan Pesantez Felipe, Vera Infante Thalia, Fernández Vélez Vanessa. Efecto anticorrosivo de pinturas de poliuretano aplicadas sobre acero galvanizado sin tratamiento superficial. Rev. del Instituto de Investigación FIGMMG-UNMSM 22(44).

[11]Menor de Gaspar Álvarez, Pedro (2020). Comportamiento estructural de uniones atornilladas de acero en función del método de mecanizado de los agujeros Máster en Ingeniería Industrial, 2020, Universidad de Cantabria URL: http://hdl.handle.net/10902/18292
[12]Flores Dávila, Fernando Alberto Análisis de la precipitación de cementita en el acero ultrabajo carbono POSCO CWA0802Tesis ESIQIE

URI: http://tesis.ipn.mx/handle/123456789/2793 9

[13] Madariaga Rosas Cristhian Harley, Modenesi Paulo José, Rincón Ortiz Mauricio. Efectos de los Active Flux sobre Soldaduras GMAW Aplicadas a un Acero AISI/SAE 1020. Soldagem \& Inspeção. 2020;25:e2502.

https://doi.org/10.1590/0104-9224/SI25.02

[14] Fernández-Columbié Tomás H., GómezFernández Maikel, Lamorú-Urgellés Marbelis, Romero-Góngora Walter, Hernández-Verdecia Maikel. (2020) Transformaciones microestructurales por efecto del calor en una unión disímil de acero 17-4 PH y Hastelloy C-276. Minería y Geología / v.36 n.1 / enero-marzo / 2020 / p. 81-91 ISSN 19938012.

[15] Ghali E., Potvin R. (1972) "The mechanism of phosphating of steel". Corrosion Science, volume 12, issue 7, pp 583-594.

[16] Alvarado Macias G. (2012). "Estudio del proceso de formación de recubrimientos de fosfato de manganeso sobre aceros", Centro de Investigaciones y de Estudios Avanzados del Instituto Politécnico Nacional Unidad Saltillo.

[17] Galvan-Reyes C., Salinas Rodriguez A., Fuentes Aceituno J.C. (2015) "Degradation and crystalline reorganization of hureaulite crystals during the manganese phosphating of a high strength steel". Surface and coatings technology, volume 275 , pages $10-$ 20. 